\section{Anxious mothers... anxious babies?}

\author{
Harriet Hiscock ${ }^{1,2,3}$
}

\author{
A day of worry is more exhausting \\ than a week of work. \\ - John Lubbock (1834-1913)
}

Many mothers of infants experience days of worry and weeks of work-an experience made so much harder by the presence of a crying infant. Up to $20 \%$ of mothers in developed countries report excessive crying in their infants. ${ }^{1}$ Although often thought of as a benign, self-limiting condition, infant crying-in particular, crying that lasts more than $3 \mathrm{~h}$ per day, for more than 3 days per week and for more than 3 weeks in a row-is associated with child abuse, early weaning and maternal depression. ${ }^{1}$

The cause(s) of excessive infant crying remains elusive. There is no doubt, however, that a mother's mood and caregiving style can impact directly on an infant's behaviour and vice versa. It is well recognised that mothers who experience postnatal depression may withdraw from their infant who, in turn, may withdraw from their mother. It is also well recognised that mothers of infants with excessive crying are more likely to report high levels of depression symptoms than mothers of infants without such crying. However, the role of maternal anxiety in infant crying has not been well researched. The article by Petzoldt et al shines a light on this largely neglected area. ${ }^{2}$ The authors highlight the unique contribution of maternal anxiety to the development of excessive crying by following a community sample of 306 mothers across seven time points from early pregnancy to 16 months postpartum. They found that mothers with an anxiety disorder prior to pregnancy were at higher risk of having a child with excessive crying at 2, 4 or 16 months postpartum compared with mothers without an anxiety disorder, and that the risk increased further for mothers who developed an anxiety disorder during pregnancy. They hypothesised that this may be due to parenting style, that is, maternal

\footnotetext{
${ }^{1}$ Community Child Health, Murdoch Childrens Research Institute, Melbourne, Victoria, Australia; ${ }^{2}$ Centre for Community Child Health, Royal Children's Hospital, Melbourne, Victoria, Australia; ${ }^{3}$ Department of Paediatrics, University of Melbourne, Victoria, Australia

Correspondence to Dr Harriet Hiscock, Community Child Health, Murdoch Children's Research Institute, Melbourne, Victoria 3052, Australia;

harriet.hiscock@rch.org.au
}

anxiety leading to intrusive parenting in turn increasing infant crying. This is a scenario often seen in the consultation room where an anxious mother tries to soothe her crying baby by constant rocking and jiggling at a time when her baby is actually tired and needs help to be settled calmly to sleep. This intrusive parenting and misreading of the baby's tired cues intensifies the baby's crying. Beyond the first year (ie, around 16 months), excessive crying may reflect a child's inability to selfsoothe and self-regulate. This could again be exacerbated by the mother's parenting. An anxious mother is more likely to be overinvolved and overprotective, ${ }^{3}$ stepping in to soothe her toddler rather than allowing him opportunities to learn to do so himself. Future research should explore whether parenting styles mediate the relationship between maternal perinatal anxiety and infant/toddler crying and the potential for interventions around this.

Another explanation for the relationship between perinatal anxiety and infant crying is the foetal programming hypothesis. Maternal anxiety during pregnancy can lead to increased maternal cortisol crossing the placenta. This may affect the development of the fetus's hypothalamic-pituitary-axis, limbic system and prefrontal cortex, leading to an infant who is more irritable and less able to self-regulate. ${ }^{4} \mathrm{~A}$ further explanation may be that a genetic predisposition to anxiety is inherited by her infant from the mother. Persistent infant crying is a risk factor for later child internalising disorders such as anxiety. ${ }^{5}$ Thus, the relationship between maternal anxiety and infant crying is probably due to a complex interplay of genetics, foetal programming and parenting.

Parenting by fathers should not be forgotten. Fathers play an increasing role in the care of their infants and can also experience anxiety disorders. Paternal antenatal and postnatal depression have been shown to uniquely contribute to later child behaviour problems, ${ }^{6}$ and paternal anxiety may play a similar role in infant crying. Future research should examine this.

Surprisingly, maternal depressive disorders experienced either before or during pregnancy did not predict maternal report of excessive infant crying. The reasons for this are unclear. It may be, as the authors suggest, that maternal depression leads to maternal withdrawal, which discourages infant crying. It may also be that mothers who are depressed are less attuned to their infant and are not aware of the extent of their infant's crying.

Petzoldt et al's study had a number of strengths. It followed a large, prospective cohort with excellent retention and used validated diagnostic interviews to ascertain maternal mental health disorders. However, as in most community-based research, women from lower socioeconomic groups were under-represented as were women from different cultural backgrounds. Whether the author's findings hold true for these groups remains to be seen.

There are some potential clinical implications of this study. The authors suggest that mothers could be potentially screened for anxiety in the perinatal period. They are rightly cautious in this suggestion as a number of criteria for screening programmes have not been met. ${ }^{7}$ For example, we do not have a clear understanding of the natural history of maternal perinatal anxiety disorders; we do not have a 'gold standard' screening test that is brief, valid and acceptable ${ }^{8}$; we do not have data to tell us when a screen should be done during the course of pregnancy and we do not know who should treat a woman with perinatal anxiety nor how best to do so.

Thus, the study by Petzoldt et al opens up a wealth of research opportunities in this complex landscape. Future research needs to replicate these findings in perinatal cohorts around the world. The roles of parenting styles, genetics the intrauterine environment, and fathers' mental health and parenting need to be further explored. In the interim, health professionals who care for women and their infants could open up a conversation around maternal anxiety and its perceived impact on the mother's parenting styles and the infant's response, provided professional support is available to address any issues arising. This needs to be done sensitively as the last thing we need to do is add to a mother's 'day of worry' by blaming her for her infant's crying.

\section{Competing interests None.}

Provenance and peer review Commissioned; internally peer reviewed.

To cite Hiscock H. Arch Dis Child 2014;99:793-794.

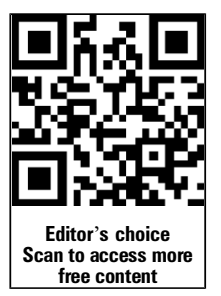


Received 6 May 2014

Revised 25 May 2014

Accepted 27 May 2014

Published Online First 19 June 2014

\section{S Linked}

- http://dx.doi.org/10.1136/archdischild-2013-305562

Arch Dis Child 2014;99:793-794.

doi:10.1136/archdischild-2014-306631

\section{REFERENCES}

1 Hiscock H, Jordan B. Problem crying in infancy. Med J Australia 2004;181:507-12.
2 Johanna P, Hans-Ulrich W, Julia W, et al. Maternal anxiety disorders predict excessive infant crying: a prospective-longitudinal cohort study. Arch Dis Child 2014;99:800-6.

3 Bayer JK, Hiscock $\mathrm{H}$, Ukoumunne OC, et al. Early childhood aetiology of mental health problems: a longitudinal population-based study. J Child Psychol Psychiatry 2008;49: 1166-74.

4 Van den Bergh BRH, Mulder EJH, Mennes M, et al. Antenatal maternal anxiety and stress and the neurobehavioural development of the fetus and child: links and possible mechanisms. A review. Neurosci Biobehav Rev 2005;29:237-58.

5 Hemmi MH, Wolke D, Schnieder S. Associations between problems With crying, sleeping and/or feeding in infancy and long-term behavioural outcomes in childhood: a meta- analysis. Arch Dis Child 2011;96:622-9.

6 Ramchandani PG, O'Connor TG, Evans J, et al. The effects of pre- and postnatal depression in fathers: a natural experiment comparing the effects of exposure to depression on offspring. J Child Psychol Psychiatry 2008;49:1069-78.

7 Wilson JMG, Jungner G. Principles and practice of screening for disease. Public Health Paper No. 34. Geneva: World Health Organization; 1968.

8 Rose M, Susan A. Anxiety measures validated in perinatal populations: a systematic review. J Affect Disord 2011;133:1-15. 